\title{
THE EFFECTS OF DUCT SHAPE ON THE NUSSELT NUMBER
}

\author{
M. Emin Erdoğan and C. Erdem Imrak \\ Faculty of Mechanical Engineering, Istanbul Technical University, 34439 \\ Gumussuyu, Istanbul, Turkey \\ imrak@itu.edu.tr
}

\begin{abstract}
This article considers the effect of duct shape on the Nusselt number. In order to show the effects of duct shape on the Nusselt number, four illustrative examples are given: the heat transfer in a duct of rectangular cross-section, heat transfer in a duct of semicircular cross-section, the heat transfer in a duct of circular crosssection and heat transfer between two parallel plates. The method used in this paper is general and it can be applied al flows. It is not necessary to use thin plate analogy, first, the velocity distribution is obtained and then the temperature distribution is found. It is shown that the Nusselt numbers calculated for the four examples cited depend not only on duct shape but also on wall friction.
\end{abstract}

Key Words- Nusselt number, duct shape, wall friction

\section{INTRODUCTION}

The effect of duct shape on heat transfer in a duct of uniform cross-section is investigated. In order to show the effect of duct shape on heat transfer, heat transfer in a duct of rectangular cross-section, heat transfer in a duct of semicircular cross-section, heat transfer in a duct of circular cross-section, and heat transfer between two parallel plates are considered. The flow and the heat transfer in these ducts, apart from their theoretical interest, are of considerable practical importance and arise frequently in industrial processes. Laminar flow forced convection in rectangular ducts has been given by Clark and Kays [1]. The heat transfer in ducts of rectangular, equilateral triangular, rightangled isoceles triangular, and semicircular cross-sections have been studied by Marco and Han [2]. They considered heat transfer in a duct of rectangular cross-section by making analogy to thin plate theory and they gave the conditions under which the solution is permissible. By this analogy, first the temperature distribution is obtained and then the velocity distribution is found. In this paper, it is shown that it is not necessary to use the thin plate analogy; first, the velocity is obtained and then the temperature distribution is found. The method used in this paper is easier than that in [2] and it can be extend to other ducts. The values of the Nusselt number in a duct of rectangular cross-section depend on the aspect ratio, $b / a$. The values of the Nusselt number for heat transfer between two parallel plates, $b / a=0$, is $140 / 17$ or about 8.23529412 to eight decimal places and for heat transfer in a duct of square crosssection, where $b / a=1$, is about 3.849172 to six decimal places. The values of the Nusselt number in [2] differ from those in this paper. Calculation of the Nusselt number in a flow in which the velocity depends on two coordinates is different from that in a 
flow in which the velocity depends on one coordinate [3].

The velocity distribution for flow in a duct of semicircular cross-section have been given by Eckert et al. [4]. They presented the results of calculation for steady laminar flow. Pressure drag and friction factor values for a duct of semicircular crosssection have been studied by Sparrow and Haji-Sheikh [5]. The linearization method was applied to developing flow in these ducts [6]. The steady flow in a curved semicircular duct has been investigated by Mashliyah [7]. Calculation of the flow was made by solving the Navier-Stokes equation numerically. The flow in a curved semicircular duct is composed of a main flow along the curved duct axis with a superimposed secondary flow. A comprehensive work on heat transfer in circular and non-circular ducts has been given in [8].

In this paper, heat transfer in ducts of rectangular cross-section, semicircular cross-section, circular cross-section and the parallel plate duct are considered. First the velocity distribution and then the temperature distribution are calculated. Using the expressions of the velocity and the temperature for the considered ducts, values of the Nusselt number are obtained. The calculation methods for velocity and temperature which depend on two coordinates are different than the methods for which they depend on only one coordinate. The value of the Nusselt number for a duct of rectangular crosssection depends on the aspect ratio, $b / a$. The value of the Nusselt number is the smallest for a duct of square cross-section for which the aspect ratio equals to unity. The value of the Nusselt number is the greatest for $b / a=0$. This value of the aspect ratio corresponds to the heat transfer between two parallel plates. The calculation in this paper is made by assuming that the surface temperature varies linearly along the duct.

In the case of a duct of semicircular cross-section the calculation of the Nusselt number is straightforward and it is found that the value of the Nusselt number is about 4.489611795 to nine decimal places. It seems to be impossible to obtain this value as a fraction.

In the case of a pipe of circular cross-section the value of the Nusselt number is $48 / 11$ or about 4.363636364 to nine decimal places. This value is smaller than that for a duct of semicircular cross-section.

In this paper, the values of the Nusselt numbers obtained are about, 3.5441771 for a duct of square cross-section; 48/11 (or 4.363636364) for a circular pipe, about 4.088184147 for a duct of semicircular cross-section and 140/17 (or 8.23529412) for a parallel plate duct. These values of the Nusselt number show the effect of duct shape on heat transfer.

\section{HEAT TRANSFER IN A DUCT OF RECTANGULAR CROSS-SECTION}

Consider the fluid in a duct of rectangular cross-section where sides are at $x= \pm a$ and $y= \pm b$ as shown in Fig. 1, it is assumed that $a \geq b$ throughout the paper. The governing equation for velocity is

$$
\frac{\partial^{2} w}{\partial x^{2}}+\frac{\partial^{2} w}{\partial y^{2}}=\frac{1}{\mu} \frac{d p}{d z}
$$


where $w(x, y)$ is the axial velocity and $d p / d z$ is the constant pressure gradient. The boundary conditions are

$$
w( \pm a, y)=0, \quad w(x, \pm b)=0 .
$$

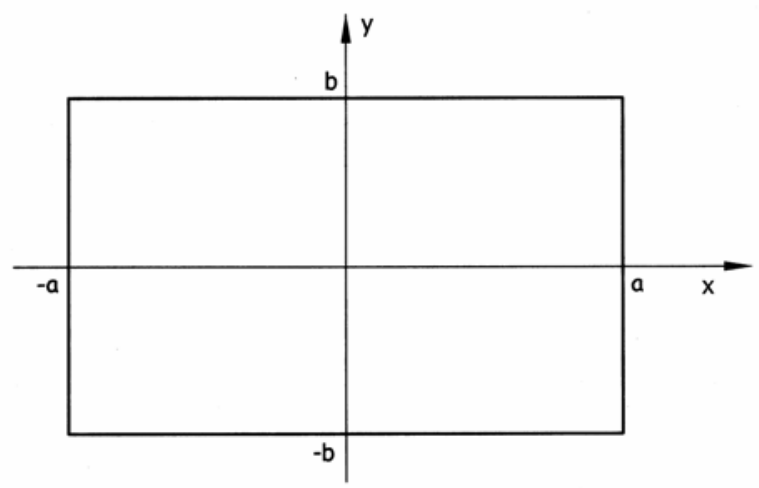

Figure 1. The coordinate system for a duct of rectangular cross-section.

The solution may be written in different forms, however, a convenient one is

$$
\frac{w}{-(1 / 2 \mu)(d p / d z) b^{2}}=\sum_{p=0}^{\infty} \sum_{q=0}^{\infty} A_{p q} \cos \mu_{p} x \cos \lambda_{q} y
$$

where $\mu_{p}=(2 p+1) \pi / 2 a$ and $\lambda_{q}=(2 q+1) \pi / 2 b$ and

$$
A_{p q}=\frac{32}{\pi^{2}} \frac{(-1)^{p+q}}{(2 p+1)(2 q+1)} \frac{1}{b^{2}\left(\mu_{p}^{2}+\lambda_{q}^{2}\right)} .
$$

When $b / a$ goes to zero equation (2) reduces to

$$
\lim _{\substack{a \\ a}} \frac{w}{-\frac{1}{2 \mu} \frac{d p}{d z} b^{2}}=\frac{32}{\pi^{3}} \sum_{q=0}^{\infty} \frac{(-1)^{q} \cos \lambda_{q} y}{(2 q+1)^{3}}=1-\frac{y^{2}}{b^{2}} .
$$

This shows the velocity between two parallel plates.

The governing equation for temperature is

$$
\frac{\partial^{2} T}{\partial x^{2}}+\frac{\partial^{2} T}{\partial y^{2}}=\frac{w}{\kappa} \frac{\partial T}{\partial z}
$$

where $T$ is temperature and $\kappa$ is thermal diffusivity. The boundary conditions for $T$ are $T=T_{w}=T_{o}+(\partial T / \partial z) z$ at the surface where $\partial T / \partial z=\alpha$ is a constant and $T=T_{0}$ at $z=0$. The solution subject to these boundary conditions is

$$
T-T_{w}=\frac{\alpha}{\kappa}\left(-\frac{1}{2 \mu} \frac{d p}{d z} b^{2}\right) \sum_{p=0}^{\infty} \sum_{q=0}^{\infty} B_{p q} \cos \mu_{p} x \cos \lambda_{q} y,
$$

where

$$
B_{p q}=\frac{32}{\pi^{2}} \frac{(-1)^{p+q}}{(2 p+1)(2 q+1)} \frac{1}{b^{2}\left(\mu_{p}^{2}+\lambda_{q}^{2}\right)} .
$$

When $b / a$ goes to zero equation (4) reduces to 


$$
\lim _{\frac{b}{a} \rightarrow 0} \frac{T-T_{w}}{-\frac{\alpha}{\kappa}\left(-\frac{1}{2 \mu} \frac{d p}{d z} b^{2}\right)}=\frac{128 b^{2}}{\pi^{5}} \sum_{q=0}^{\infty} \frac{(-1)^{q} \cos \lambda_{q} y}{(2 q+1)^{5}}=\frac{b^{2}}{2}\left[\left(1-\frac{y^{2}}{b^{2}}\right)-\frac{1}{b}\left(1-\frac{y^{4}}{b^{4}}\right)\right] .
$$

This is the temperature distribution between two parallel plates.

The Nusselt number is the ratio of the convective conductance to the pure molecular conductance and is defined as

$$
N u=\frac{q}{k\left(T_{w}-T_{M}\right) S / D},
$$

where $q$ is the quantity of heat transferred per unit time from an immersed body through an area $S, T_{w}-T_{M}$ is a representative temperature difference, $D$ is the hydraulic diameter or a representative length and $T_{M}$ is the mean temperature weighted with respect to the velocity given in the following form

$$
T_{M}=\frac{\int_{A} T w d A}{\int_{A} w d A}
$$

where integrals are surface integrals and

$$
T_{w}-T_{M}=\frac{\int_{A}\left(T_{w}-T\right) w d A}{\int_{A} w d A}
$$

and

$$
q=\rho c \int_{A} w \frac{\partial T}{\partial z} d A .
$$

Hence inserting this expression, the Nusselt number can be written as [2]

$$
N u=-\frac{D \rho c \int_{A} w \frac{\partial T}{\partial z} d A}{S k \int_{A} w\left(T-T_{w}\right) d A / \int_{A} w d A} .
$$

$D / S$ is the ratio of the hydraulic diameter to the perimeter of the cross-section times unit length. Hydraulic diameter is defined as four times the wetted area to the perimeter of the cross-section. Using the surface temperature, equation (5) becomes

$$
N u=-\frac{D \rho c \alpha}{S k} \frac{\left(\int_{A} w d A\right)^{2}}{\int_{A} w\left(T-T_{w}\right) d A} .
$$

Inserting the expressions for $w$ and $\left(T-T_{w}\right)$ and evaluating the surface integrals, one finds

$$
N u=\frac{64 / \pi^{2}}{\left(1+\frac{b}{a}\right)^{2}} \frac{\left[\sum_{p=0}^{\infty} \sum_{q=0}^{\infty} \frac{1}{(2 p+1)^{2}(2 q+1)^{2}} \frac{1}{(2 p+1)^{2}\left(b^{2} / a^{2}\right)+(2 q+1)^{2}}\right]^{2} \sum_{p=0}^{\infty} \frac{1}{(2 p+1)^{2}(2 q+1)^{2}} \frac{1}{\left[(2 p+1)^{2}\left(b^{2} / a^{2}\right)+(2 q+1)^{2}\right]^{3}}}{.}
$$

$b / a=0$ corresponds to the value of the Nusselt number between two parallel plates and since 


$$
\sum_{p=0}^{\infty} \frac{1}{(2 p+1)^{2}}=\frac{\pi^{2}}{8} \quad \text { and } \quad \sum_{q=0}^{\infty} \frac{1}{(2 q+1)^{4}}=\frac{\pi^{4}}{96},
$$

one finds $N u=140 / 17$. This value is verified by calculating the Nusselt number for a duct of two parallel plates which is about 8.235294118 to nine decimal places. However, this value is given in [2] as about 8.21. $b / a=1$ corresponds to a duct of square cross-section and the value of the Nusselt number is about 3.5491771 to seven decimal places. However, this value in [2] is about 3.60. The variation of the Nusselt number with respect to $b / a$ is illustrated in Fig. 2 . It is clearly seen that when $b / a$ tends to zero, the Nusselt number reduces to that for the Nusselt number between two parallel plates and when $b / a$ is equal to unity, the Nusselt number reduces to that for the Nusselt number in a duct of square cross-section. The value of the Nusselt number is the greatest for $b / a=0$ and the smallest for $b / a=1$.

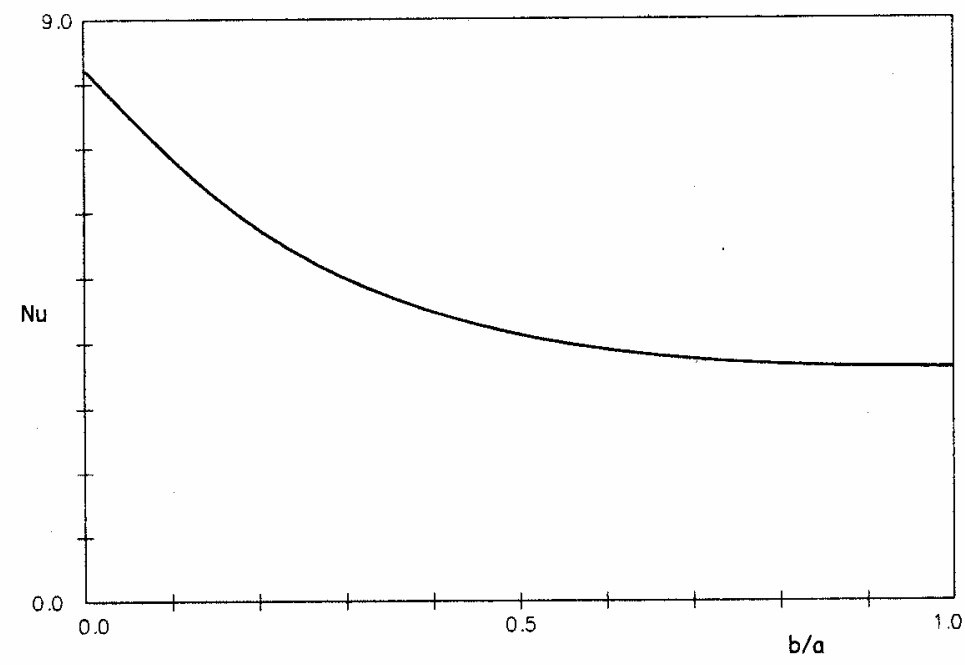

Figure 2. The variation of the $N u$ number with respect to the aspect ratio, $b / a$.

\section{HEAT TRANSFER IN A DUCT OF SEMICIRCULAR CROSS-SECTION}

Consider the fluid in a duct of semicircular cross-section and the flow geometry is shown in Fig. 3. The governing equation for velocity is

$$
\frac{\partial^{2} w}{\partial r^{2}}+\frac{1}{r} \frac{\partial w}{\partial r}+\frac{1}{r^{2}} \frac{\partial^{2} w}{\partial \theta^{2}}=\frac{1}{\mu} \frac{d p}{d z},
$$

where $w(r, \theta)$ is the axial velocity and $d p / d z$ is the constant pressure gradient. The boundary conditions are

$$
w(a, \theta)=0, \quad w(r, 0)=0, \quad w(r, \pi)=0 .
$$




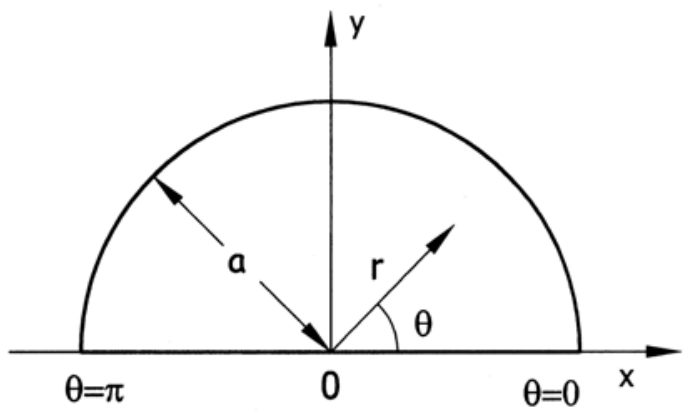

Figure 3. Flow geometry for a duct of semicircular cross-section.

The solution of the governing equation subject to the boundary conditions is

$$
w(r, \theta)=\sum_{n=0}^{\infty} f_{2 n+1}(r) \sin (2 n+1) \theta,
$$

where

$$
f_{2 n+1}(r)=-\frac{1}{\mu} \frac{d p}{d z} \frac{(4 / \pi) a^{2}}{(2 n-1)(2 n+1)(2 n+3)}\left(\frac{r^{2}}{a^{2}}-\frac{r^{2 n+1}}{a^{2 n+1}}\right) .
$$

The governing equation for temperature is

$$
\frac{\partial^{2} T}{\partial r^{2}}+\frac{1}{r} \frac{\partial T}{\partial r}+\frac{1}{r^{2}} \frac{\partial^{2} T}{\partial \theta^{2}}=\frac{w}{\kappa} \frac{\partial T}{\partial z},
$$

where $T$ is temperature and $\kappa$ is thermal diffusivity. The boundary conditions for $T$ are $T=T_{w}=T_{o}+(\partial T / \partial z) z$ at the surface where $\partial T / \partial z=\alpha$ is a constant and $T=T_{0}$ at $z=0$. The solution of equation (10) is

$$
T-T_{w}=\sum_{n=0}^{\infty} f_{2 n+1}(r) \sin (2 n+1) \theta,
$$

where

$$
\begin{aligned}
f_{2 n+1}(r) & =\frac{1}{\mu} \frac{d p}{d z} \frac{\alpha(4 / \pi) a^{4}}{\kappa} \frac{1}{(2 n-1)(2 n+1)(2 n+3)}\left[\frac{1}{(2 n-3)(2 n+5)}\left(\frac{r^{4}}{a^{4}}-\frac{r^{2 n+1}}{a^{2 n+1}}\right)\right. \\
& \left.-\frac{1}{8(n+1)}\left(\frac{r^{2 n+1}}{a^{2 n+1}}-\frac{r^{2 n+3}}{a^{2 n+3}}\right)\right] .
\end{aligned}
$$

The Nusselt number is defined by equation (5). For a duct of semicircular crosssection $D / S=2 \pi /(\pi+2)^{2}$. Inserting the expressions for $w$ and $T-T_{w}$ into equation (6) and evaluating of the surface integrals, one finds

$$
N u=\frac{1}{256} \frac{\left(\pi^{2}-8\right)^{2}}{(\pi+2)^{2}} \frac{1}{\sum_{n=0}^{\infty} F_{n}}
$$

where 


$$
\begin{aligned}
F_{n}= & -\frac{1}{4(n+1)(2 n-3)(2 n-1)(2 n+1)^{2}(2 n+3)^{2}(2 n+5)^{2}} \\
& +\frac{1}{8(2 n-3)(2 n-1)(2 n+1)^{2}(2 n+3)^{2}(2 n+5)(2 n+7)} \\
& -\frac{1}{32(n+1)^{2}(2 n-1)(2 n+1)^{2}(2 n+3)^{2}(2 n+5)} \\
& +\frac{1}{16(n+1)(2 n-1)(2 n+1)^{2}(2 n+3)^{3}(2 n+7)} .
\end{aligned}
$$

The value of the Nusselt number is about 4.088184147 to nine decimal places. This value of the Nusselt number is comparable to that for a pipe of circular crosssection.

\section{HEAT TRANSFER IN A PIPE OF CIRCULAR CROSS-SECTION}

Consider the fluid in a pipe of circular cross-section as shown in Fig. 4. The governing equation for velocity is

$$
\frac{\partial^{2} w}{\partial r^{2}}+\frac{1}{r} \frac{\partial w}{\partial r}=\frac{1}{\mu} \frac{d p}{d z},
$$

where $w(r)$ is the axial velocity and $d p / d z$ is the constant pressure gradient. The boundary condition is $w(a)=0$, where a is the radius of the pipe. The solution of the governing equation satisfying the boundary condition and the uniformity at $r=0$ is

$$
w=w_{0}\left(1-\frac{r^{2}}{a^{2}}\right)
$$

where $w_{0}$ is the velocity at $r=0$.

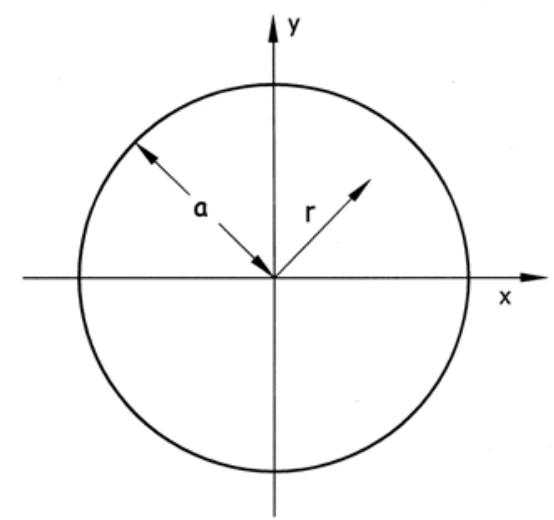

Figure 4. Flow geometry for flow in a circular pipe.

The governing equation for temperature is

$\frac{\partial^{2} T}{\partial r^{2}}+\frac{1}{r} \frac{\partial T}{\partial r}=\frac{w}{\kappa} \frac{\partial T}{\partial z}$. 
The boundary conditions for $T$ are $T=T_{w}=T_{o}+(\partial T / \partial z) z$ at the surface where $\partial T / \partial z=\alpha$ is a constant and $T=T_{0}$ at $z=0$. The solution of equation (13) is

$$
T-T_{w}=\frac{\alpha w_{0}}{16 \kappa} a^{2}\left(4 \frac{r^{2}}{a^{2}}-\frac{r^{4}}{a^{4}}-3\right) .
$$

The maximum temperature is at the center of the pipe. The Nusselt number is defined by equation (5). For a pipe of circular cross-section $D / S=1 / \pi$. Since $\partial T / \partial z=\alpha$ is a constant, equation (5) can be written as

$$
N u=-\frac{D \rho c \alpha}{S k} \frac{\left(\int_{A} w d A\right)^{2}}{\int_{A} w\left(T-T_{w}\right) d A} .
$$

Inserting the expressions for $w$ and $T-T_{w}$ into this expression and performing the surface integrals, one finds $N u=48 / 11$ or about 4.363636364 to nine decimal places. It is clearly seen that the value of the Nusselt number for a duct of semicircular crosssection is larger than that for a pipe of circular cross-section.

\section{HEAT TRANSFER BETWEEN TWO PARALLEL PLATES}

Consider the fluid between two parallel plates as shown in Fig. 5. The governing equation for velocity is

$$
\frac{d^{2} u}{d y^{2}}=\frac{1}{\mu} \frac{d p}{d x}
$$

where $u(y)$ is the velocity and $d p / d x$ is the constant pressure gradient. The boundary condition is $u( \pm b)=0$. The solution of the governing equation is $u=u_{0}\left[1-\left(y^{2} / b^{2}\right)\right]$, where $u_{0}$ is the velocity at $y=0$.

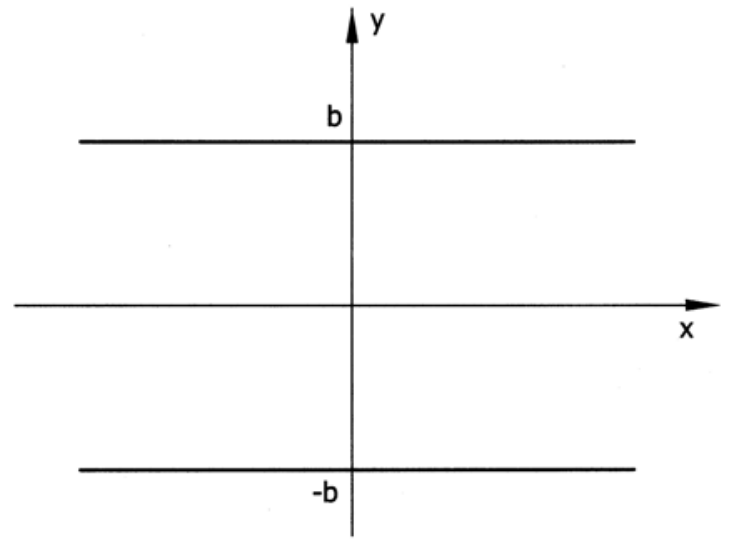

Figure 5. Flow geometry for flow between two parallel plates.

The governing equation for temperature is

$$
\frac{d^{2} T}{d y^{2}}=\frac{u}{\kappa} \frac{d T}{d x} .
$$


The boundary conditions for $T$ are $T=T_{u}=T_{0}+(\partial T / \partial x) x$ at the surface where $d T / d x=\alpha$ is a constant, and $T=T_{0}$ at $x=0$. The solution subject to the condition at the surface is

$$
T-T_{w}=-\frac{\alpha u_{0} b^{2}}{2 \kappa}\left[\left(1-\frac{y^{2}}{b^{2}}\right)-\frac{1}{6}\left(1-\frac{y^{4}}{b^{4}}\right)\right] .
$$

The Nusselt number is defined by equation (5). For a parallel plate duct $D / S$ is $2 b$. Since $d T / d x=\alpha$ is a constant, equation (5) can be written as

$$
N u=-\frac{D \rho c \alpha}{S k} \frac{\left(\int_{A} u d A\right)^{2}}{\int_{A} u\left(T-T_{w}\right) d A} .
$$

Inserting the expressions for $u$ and $T-T_{w}$ into this expression and evaluating the surface integrals, one finds $N u=140 / 17$ or about 8.235294118 to nine decimal places. This value of the Nusselt number is the highest for the examples considered.

\section{CONCLUSIONS}

The effect of duct shape on the Nusselt number is investigated. In order to show the effect of duct shape, four illustrative examples are given. One of them is heat transfer in a duct of rectangular cross-section, the second is heat transfer in a duct of semicircular cross-section, the third is heat transfer in a pipe of circular cross-section and the fourth is heat transfer between two parallel plates. The methods used in this paper can be applied to all flows. It is necessary to use the thin plate analogy. First, the velocity distribution is obtained and then the temperature distribution is found. The values of the Nusselt numbers are: about 3.5491172 for a duct of square cross-section, about 4.363636364 for a circular pipe, about 4.088184147 for a duct of semicircular cross-section and about 8.235294118 for a flat duct. Values of the Nusselt numbers obtained in the four examples considered show clearly that the Nusselt number depends on the duct shape.

Acknowledgment-The authors thank Professor Mehmet Pakdemirli and the refree.

\section{REFERENCES}

1. S.H. Clark and W.M. Kays, Laminar flow forced convection in rectangular tubes, Trans. ASME 75, 859-866, 1953.

2. S.M. Marco and L.S. Han, A note on limiting laminar Nusselt number in ducts with constant temperature gradient by analogy to thin plate theory, Trans. ASME 77, 625630, 1955.

3. S. Goldstein (Ed.), Modern Developments in Fluid Dynamics,Vol.2, second edn., Roder Pub., New York, 1965.

4. E.R.G. Eckert, T.P.Jr. Irwin and J.T. Yen, Local laminar heat transfer in wedgeshaped passages, Trans. ASME 80, 1433-1438, 1958.

5. E.R. Sparrow and A. Haji-Sheikh, Laminar heat transfer and pressure drag in isoceles 
triangular, right triangular and circular sector ducts, Trans. ASME 87, 426-427, 1965.

6. H.M. Soliman, B.B. Munis and B.C. Trap, Laminar flow in the entrance region of circular sector ducts, Trans. ASME, J. Appl. Mech. 49, 640-642, 1982.

7. J.H. Mashliyah, On laminar flow in curved semicircular ducts, J. Fluid. Mech. 99, 469-479, 1980.

8. S. Kakaç, R.K. Shah and W. Anng (Eds), Handbook of single-phase convective heat transfer, John Wiley \& Sons, New York, 1987. 